\title{
Management of chronic hepatitis C treatment failures: role of consensus interferon
}

\author{
Stevan A Gonzalez' \\ Emmet B Keeffe ${ }^{2}$ \\ 'Division of Hepatology, Baylor \\ Regional Transplant Institute, Baylor \\ All Saints Medical Center, Fort \\ Worth and Baylor University Medical \\ Center, Dallas, TX, USA; ' ${ }^{2}$ Division of \\ Gastroenterology and Hepatology, \\ Department of Medicine, Stanford \\ University Medical Center, Stanford, \\ CA, USA
}

\begin{abstract}
A significant proportion of patients with chronic hepatitis $\mathrm{C}$ virus (HCV) infection who undergo antiviral therapy have persistent or recurrent viremia and fail to achieve a sustained virologic response (SVR). Factors associated with treatment failure include HCV genotype 1 infection, high serum HCV RNA levels, and advanced fibrosis. Consensus interferon (CIFN) is a synthetic type I interferon derived from a consensus sequence of the most common amino acids found in naturally occurring alpha interferon subtypes. Several prospective clinical studies have demonstrated that CIFN may be a treatment option in patients who have failed prior interferonbased therapy, including those who have failed combination therapy with standard interferon or peginterferon plus ribavirin. Daily CIFN in combination with ribavirin may be an effective regimen in this setting; however, optimal dose and treatment duration of CIFN therapy have not been well established. Patients who achieve viral suppression during prior interferon-based therapy and those who do not have advanced fibrosis have a greater likelihood of achieving a SVR with CIFN retreatment. Individualized therapy targeting specific patient groups will be an important consideration in the successful management of prior treatment failures. Additional prospective studies are required in order to identify optimal treatment strategies for the use of CIFN in these patients.
\end{abstract}

Keywords: consensus interferon, hepatitis $\mathrm{C}$, interferon, nonresponder, relapser

\section{Introduction}

Hepatitis $\mathrm{C}$ virus (HCV) infection is the most common cause of newly diagnosed chronic liver disease in the United States, accounting for up to two-thirds of all cases. ${ }^{1}$ Approximately $20 \%$ of chronic HCV-infected individuals may subsequently develop cirrhosis, ${ }^{2}$ resulting in an increased risk of progressive liver failure and hepatocellular carcinoma (HCC). Antiviral therapy is an important consideration in the management of patients with chronic $\mathrm{HCV}$ infection, as a sustained virologic response (SVR) to therapy may halt fibrosis progression, decrease the risk of HCC, and improve survival. ${ }^{3-5}$

Treatment regimens for chronic hepatitis $\mathrm{C}$ have evolved over the last 12 years, resulting in improved SVR rates. Despite these advances, the current gold standard therapy with peginterferon alfa-2a or alfa-2b (PEG IFN) and ribavirin (RBV) is successful in only one-half of treated patients. ${ }^{6,7}$ Various clinical and virologic factors are well established as predictors of a reduced response to interferon-based therapy, including genotype 1, high baseline serum HCV RNA level, obesity, African American ethnicity, older age, and presence of advanced fibrosis or cirrhosis ${ }^{6-9}$ Therefore, a significant proportion of treated patients, particularly those infected with HCV genotype 1, will have persistent or recurrent viremia in spite of therapy and are potential candidates for retreatment.

Consensus interferon (CIFN) is a synthetic recombinant type I interferon protein derived from a consensus sequence of the most common amino acids found in naturally occurring alpha interferon subtypes. Although CIFN is not considered a first-line agent in the treatment of naïve patients with chronic hepatitis $\mathrm{C}$, it may have a potential role 
in the management of patients who previously failed prior interferon-based therapy.

\section{Standard of care with interferon-based therapy}

Since the initial studies utilizing standard interferon alpha (IFN) monotherapy in the treatment of chronic HCV infection, ${ }^{10}$ interferon-based therapy has proven to be effective in suppressing HCV RNA to undetectable levels on treatment and achieving persistent undetectability 6 months after completion of therapy, ie, an SVR. The addition of ribavirin was subsequently demonstrated in prospective clinical trials to result in improved outcomes with higher SVR rates compared to that achieved with IFN monotherapy. ${ }^{11,12}$ A further improvement was the replacement of standard IFN with PEG IFN, which, in combination with RBV, ${ }^{6,7}$ is the most effective antiviral regimen in naïve patients and the current standard of care. ${ }^{13}$ PEG IFN plus RBV is associated with an SVR of up to $52 \%$ in genotype 1 patients following a 48 -week course of therapy and $81 \%$ in genotype 2 and 3 patients following a 24-week course of therapy. ${ }^{6-8}$ An SVR is a durable response and indicates eradication of $\mathrm{HCV}$, with documented long-term undetectable serum HCV RNA in up to 18 years of follow-up. ${ }^{5}$

Although these results are gratifying, a significant proportion of patients, particularly those infected with $\mathrm{HCV}$ genotype 1, fail antiviral therapy. Treatment failure is defined as the presence of detectable serum HCV RNA 6 months following the completion of therapy, although failure may be recognized earlier in the course of therapy. Patients undergoing antiviral therapy may develop breakthrough viremia following a period of undetectable HCV RNA during a course of therapy, relapse of viremia following completion of therapy, or may be considered "null" responders with persistent viremia throughout their course of treatment. Due to a negative predictive value approaching $100 \%$ in patients who fail to achieve an early virologic response (EVR, defined by at least a $2-\log _{10}$ decrease in HCV RNA by week 12), these patients are considered nonresponders and therapy is discontinued at this time point. ${ }^{7,14}$ Additionally, any patients with detectable HCV RNA after 24 weeks of therapy will not achieve an SVR, and thus are also classified as nonresponders.

\section{Peginterferon in the retreatment of chronic HCV}

Peginterferon in combination with RBV may be considered as a treatment option in patients who failed prior IFN therapy.
Several prospective studies have found that nonresponders to prior courses of standard IFN monotherapy and relapsers to standard IFN with or without RBV may derive the most benefit from retreatment with PEG IFN and RBV. ${ }^{15-22}$ Prior nonresponders to standard IFN monotherapy achieve SVR rates ranging from $21 \%$ to $28 \%$ with PEG IFN and RBV treatment, ${ }^{15-19}$ while relapsers to combination standard IFN and RBV have been reported to achieve SVR in 38\% to 58\% of cases with PEG IFN and RBV. ${ }^{16-21}$ In contrast, combination therapy null responders to IFN and RBV tend to demonstrate relatively poor responses to retreatment with PEG IFN and RBV, with an SVR ranging from $8 \%$ to $15 \%,{ }^{15-17,21}$ but up to $20 \%$ in patients with lower baseline HCV RNA levels..$^{18,19,22}$ Based on these studies, patients with low baseline HCV RNA levels or mild to moderate fibrosis appear to have a greater potential for a SVR. ${ }^{15,16,18,21,22}$ In patients who are PEG IFN nonresponders, there is virtually no role for retreatment with repeated courses of PEG IFN, as these patients have demonstrated a resistance to this form of therapy and would likely have a negligible potential for achieving SVR. Altogether, given these relatively low retreatment response rates, there is a need for alternative therapies in previous nonresponders to IFN-based therapy. Thus, CIFN may be a treatment option in selected patients with chronic hepatitis $\mathrm{C}$ who have failed previous IFN-based therapy.

\section{Consensus interferon}

Type 1 interferons, notably IFN- $\alpha$ and IFN- $\beta$, play a critical role in the host antiviral response to HCV through inhibitory effects on viral production and activation of cellular immunity and clearance of infected cells. ${ }^{23}$ Activation of transcription factors within infected cells leads to a transcriptional response promoting the secretion of type 1 IFNs and subsequent activation of the Janus kinase-signal transducer and activator of transcription (JAK-STAT) signal transduction pathway. This process results in transcriptional activity within infected cells that promotes inhibition of $\mathrm{HCV}$ replication through the disruption of viral RNA translation and RNA synthesis. These events induce an antiviral state which limits cell-to-cell virus spread and primes immune effector cells involved in host adaptive immunity. ${ }^{23}$

In addition to having an important role in host endogenous antiviral responses to HCV, IFNs, particularly IFN- $\alpha$, have demonstrated clinical efficacy in the treatment of chronic $\mathrm{HCV}$ infection. In contrast to IFN- $\beta$ which is derived from a single gene encoding for a specific protein, IFN- $\alpha$ is a family of proteins with over a dozen subtypes differing in their respective amino acid sequences. ${ }^{24} \mathrm{CIFN}$ is a synthetic protein 
engineered by the creation of a consensus sequence involving the most common amino acids found in naturally occurring alpha interferon subtypes. In vitro studies have suggested that CIFN may have more enhanced biologic activity and increased antiviral efficacy compared with naturally occurring IFN- $\alpha$ subtypes such as IFN alfa- $2 a$ and IFN alfa- $2 b{ }^{25}$

Various dosing regimens of CIFN have been studied, although the optimal dose in naïve patients or in the setting of retreatment has not been well established. The recommended dosing based on initial phase III clinical trials is $9 \mu \mathrm{g} 3$ times weekly in treatment-naïve patients and $15 \mu \mathrm{g} 3$ times weekly in nonresponders. ${ }^{26}$ One prospective randomized trial found no improvement in SVR with higher doses CIFN at $18 \mu \mathrm{g}$ versus $9 \mu \mathrm{g}$ when given 3 times weekly in combination with RBV. ${ }^{27}$ Additionally, high doses of CIFN may be associated with a greater need for dose reductions due to adverse events. ${ }^{27}$ The rate of SVR associated with CIFN therapy could potentially be further improved with daily dosing, as this may achieve optimal viral suppression and similar kinetics to long-acting PEG IFN, resulting in a greater virologic response. ${ }^{28}$ As a result, recent studies have explored the role of daily CIFN therapy, which we will discuss in greater detail below. Table 1 summarizes the various clinical trials that have evaluated CIFN in the retreatment of prior therapy failures in patients with chronic HCV infection, while Figures 1 and 2 depict reported rates of SVR in prior treatment relapsers and nonresponders, respectively.

\section{Consensus interferon in naïve patients with chronic hepatitis $C$}

Initial clinical trials of CIFN monotherapy as a treatment for chronic hepatitis $\mathrm{C}$ in naïve patients at a dose of $9 \mu \mathrm{g}$ demonstrated greater reduction in serum HCV RNA and comparable long-term efficacy compared with standard IFN alfa- $2 \mathrm{~b}$ when given 3 times weekly. ${ }^{29}$ Subsequent prospective studies suggested that CIFN may have a significant advantage over standard IFN, particularly for difficult-to-treat patients with higher baseline serum HCV RNA levels and those infected with HCV genotype 1. ${ }^{30,31}$ The development of PEG IFN alfa-2a and alfa- $2 b$ has resulted in the ability to achieve a substantially higher rate of SVR in naïve patients ${ }^{6,7}$ and at this time, a pegylated formulation of CIFN is not available. Although one recent study revealed that CIFN 3 times weekly in combination with weight-based RBV may be as effective as combination PEG IFN alfa-2b and weight-based RBV in the treatment of HCV genotype 1 patients, comparative data are limited. ${ }^{32}$ Therefore CIFN is not considered the standard of care for naïve patients with chronic HCV infection.

\section{Consensus interferon in the retreatment} of chronic hepatitis C

\section{Consensus interferon monotherapy}

As initial attempts at IFN-based therapy for the treatment of chronic hepatitis $\mathrm{C}$ were associated with high rates of nonresponse and relapse, CIFN was introduced as an alternative therapy for patients who failed prior courses of standard IFN alfa-2a or alfa-2b in the hopes of achieving an SVR with retreatment. An early study was notable for SVR rates of $13 \%$ and $58 \%$ for nonresponders and relapsers, respectively, following a course of 48 weeks of CIFN monotherapy at $15 \mu \mathrm{g} 3$ times weekly in those who had previously received only 24 weeks of either IFN alfa- $2 \mathrm{~b}$ or lower-dose CIFN (3 $\mu \mathrm{g} 3$ times weekly). ${ }^{33}$ Further analysis of this study suggested that nonresponder patients who managed to achieve viral clearance during prior therapy followed by virologic breakthrough had a greater likelihood of SVR with CIFN retreatment. ${ }^{34}$ Similar SVR rates have been reported in IFN monotherapy relapsers with daily dosing of CIFN over a 24-week course, with a significantly greater frequency of SVR compared with combination IFN and RBV. ${ }^{35}$ CIFN monotherapy has also been studied in genotype 1 patients who failed prior therapy with combination standard IFN and RBV. SVR rates of up to $33 \%$ and $42 \%$ have been reported for combination nonresponders and relapsers treated with CIFN monotherapy, respectively, with a greater likelihood of SVR seen in patients with low baseline HCV RNA levels and minimal fibrosis. ${ }^{36}$ However, lower response rates have also been reported in this population ${ }^{37}$ and CIFN monotherapy may not be sufficient to achieve SVR in patients who were null responders to combination therapy with RBV.

\section{Consensus interferon in combination with ribavirin}

As in the case of IFN alfa-2a and alfa-2b therapy, many prospective studies have reported a greater likelihood of achieving SVR in the retreatment of prior therapy failures when combining CIFN with RBV as opposed to CIFN monotherapy. Consequently, in order to provide optimal viral suppression, more recent studies have evaluated the role of CIFN in combination with RBV as retreatment for chronic hepatitis $\mathrm{C}$. Based on the results from trials involving CIFN as monotherapy, various dosing regimens have been used, initially with CIFN administered 3 times weekly or daily dosing. Several prospective randomized trials have also evaluated the use of high-dose induction therapy with CIFN early during a course of retreatment; ${ }^{38-40}$ however, it appears that this strategy might not have a significant impact on SVR, as noted in treatment-naïve patients who received CIFN. ${ }^{41}$ 


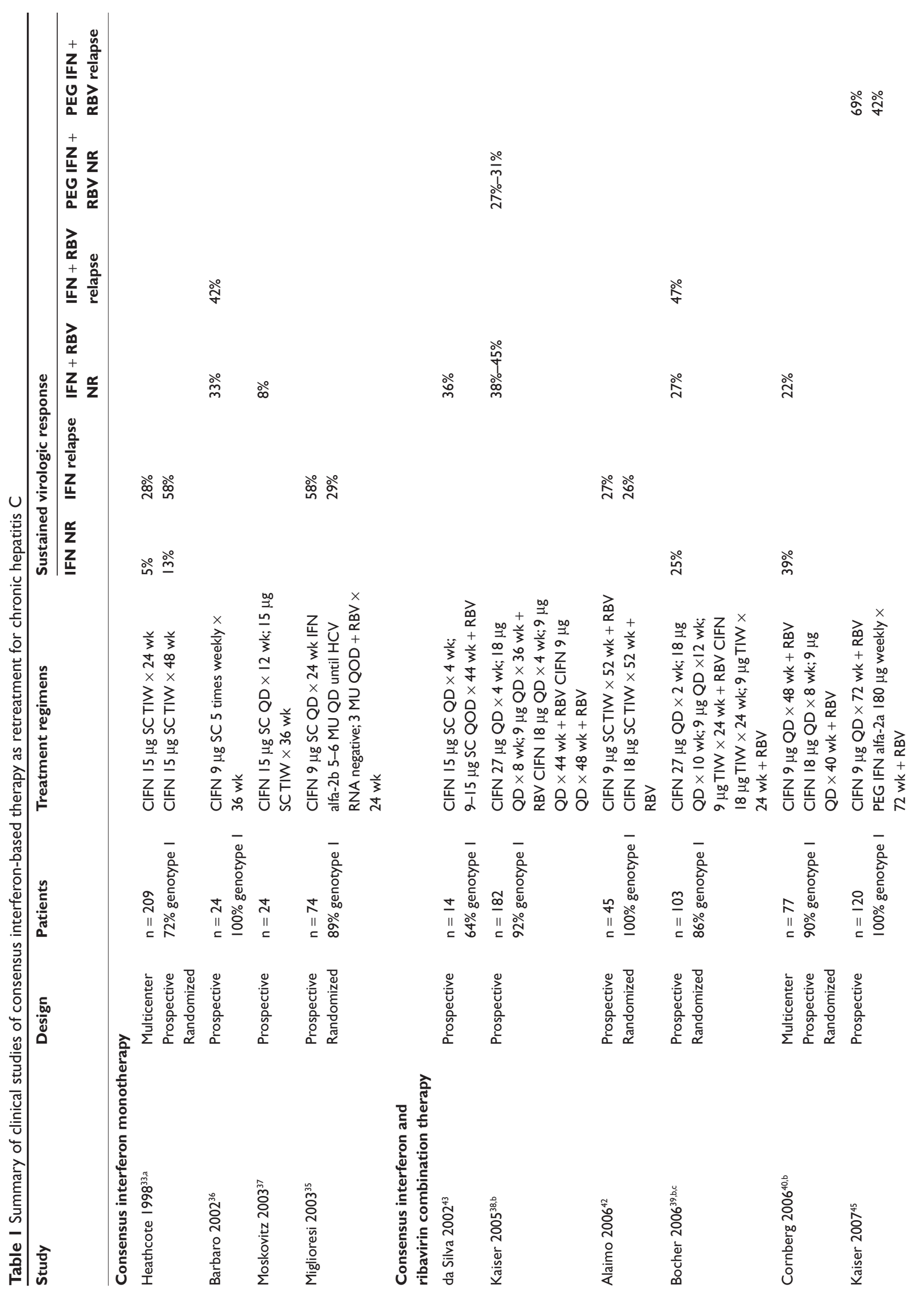


Interferon monotherapy failure

Patients who were null responders or relapsed following a course of standard IFN monotherapy might be ideal candidates for retreatment, as they might benefit from the addition of RBV to IFN-based therapy. Two prospective European trials reported a significant improvement in SVR rates in standard IFN monotherapy nonresponders when retreated with CIFN and RBV combination therapy. ${ }^{39,40}$ Both of these studies included at least 24 weeks of daily CIFN, and the SVR rate reached $39 \%$ with daily CIFN treatment over a 48-week course. ${ }^{40}$ Another European study evaluated CIFN 3 times weekly plus RBV in previous relapsers to standard IFN monotherapy ${ }^{42}$ Of note, this trial reported discontinuation due to adverse events in over one quarter of patients enrolled, which may have resulted in a lower SVR than expected.

\section{Combination interferon and ribavirin failure}

CIFN and RBV therapy for prior nonresponders to combination standard IFN plus RBV appears to result in SVR rates that are comparable to, and in some cases exceed, those that have been reported with PEG IFN plus RBV retreatment regimens. ${ }^{38-40,43}$ The most impressive response rates have emerged from two studies that reported an SVR ranging from $36 \%$ to $45 \% .^{38,43}$ Although one study had a relatively small sample size, the study with the highest reported SVR by Kaiser and colleagues enrolled 121 combination IFN/RBV nonresponders. ${ }^{37}$ These prospective studies included a majority of patients with genotype 1 infection and up to approximately one-third with advanced fibrosis on liver biopsy. Combination IFN plus $\mathrm{RBV}$ relapsers may potentially have an even greater virologic response to CIFN-based therapy, although this population has not been studied as extensively. One randomized clinical trial by Bocher and colleagues, which combined one-third IFN relapsers and two-thirds IFN/RBV relapsers, reported a rate of SVR up to $70 \%$ in a treatment arm that received daily CIFN at induction doses up to $27 \mu \mathrm{g}$ subcutaneously per day with ongoing daily therapy for 24 weeks followed by dosing 3 times weekly for the remainder of the 48 -week course. ${ }^{39}$ However, due to a relatively low number of patients, the results were not statistically different from a noninduction arm with a SVR of $38 \%$. Taken together, the overall SVR of relapsers in this trial was $47 \%$.

\section{Combination peginterferon and ribavirin failure}

The most challenging population of patients who might benefit from retreatment is patients who have failed prior combination therapy with the current gold standard of PEG 


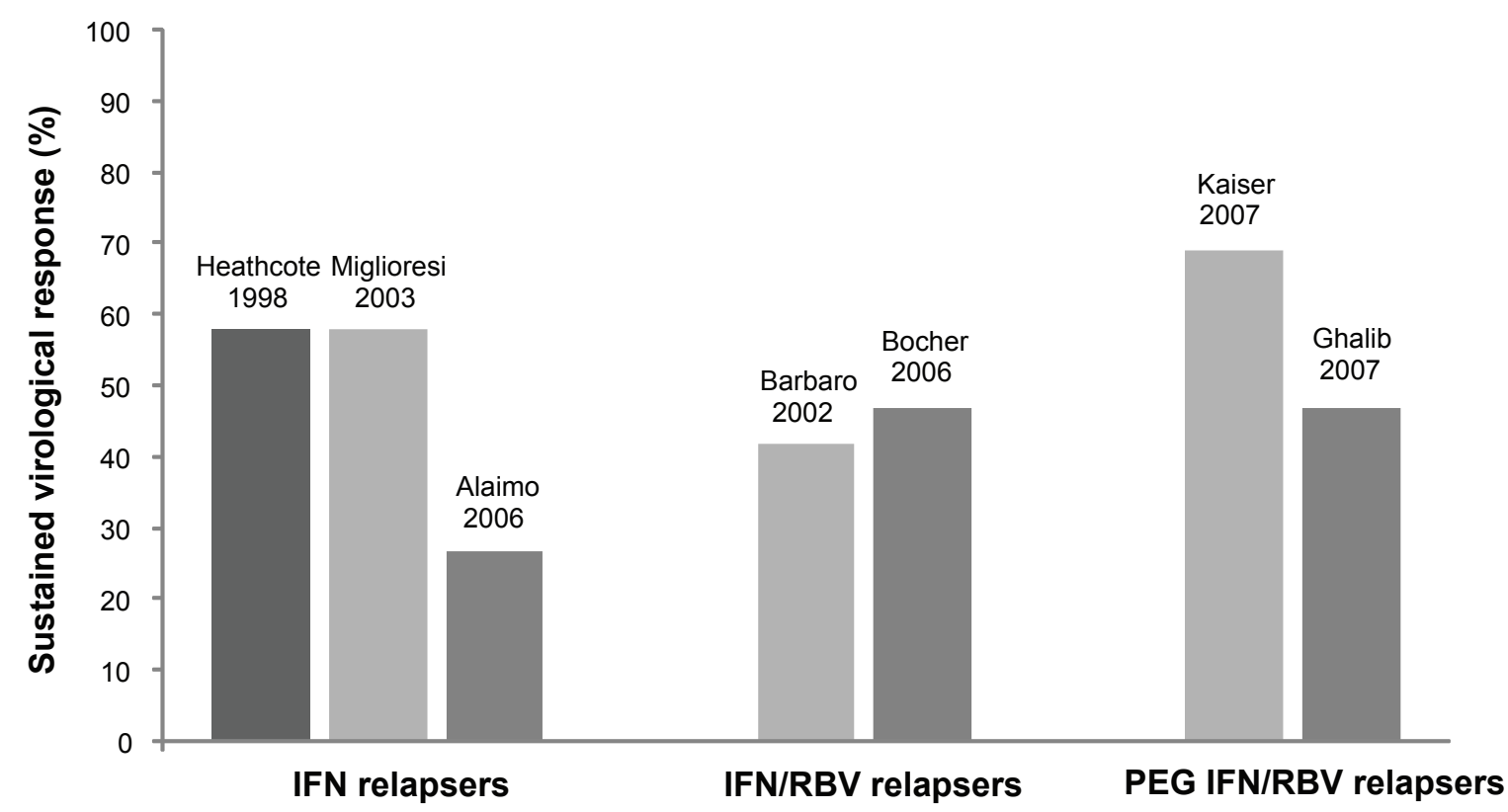

Figure I Clinical studies of consensus interferon in the retreatment of prior relapsers to interferon-based therapy. ${ }^{\mathrm{a}}$

${ }^{a}$ Note the studies by Heathcote et $\mathrm{a}^{33}$ Miglioresi et $\mathrm{a}^{35}$ and Barbaro et $\mathrm{a}^{36}$ used consensus interferon as monotherapy. The remaining studies used combination consensus interferon and ribavirin.

Abbreviations: IFN, interferon; PEG IFN, peginterferon; RBV, ribavirin.

IFN and RBV. In this population with limited options for retreatment, CIFN might have an important role in successful clearance of HCV infection. Particularly those who have relapsed to prior PEG IFN and RBV may derive the most benefit from retreatment with CIFN. One retrospective study by Ghalib et $\mathrm{al}^{44}$ and one prospective study with an extended 72-week course by Kaiser et $\mathrm{al}^{45}$ reported SVR rates of $47 \%$ and $69 \%$, respectively, with daily CIFN plus RBV. Kaiser and colleagues also noted that daily CIFN plus RBV was superior to retreatment with combination PEG IFN and RBV

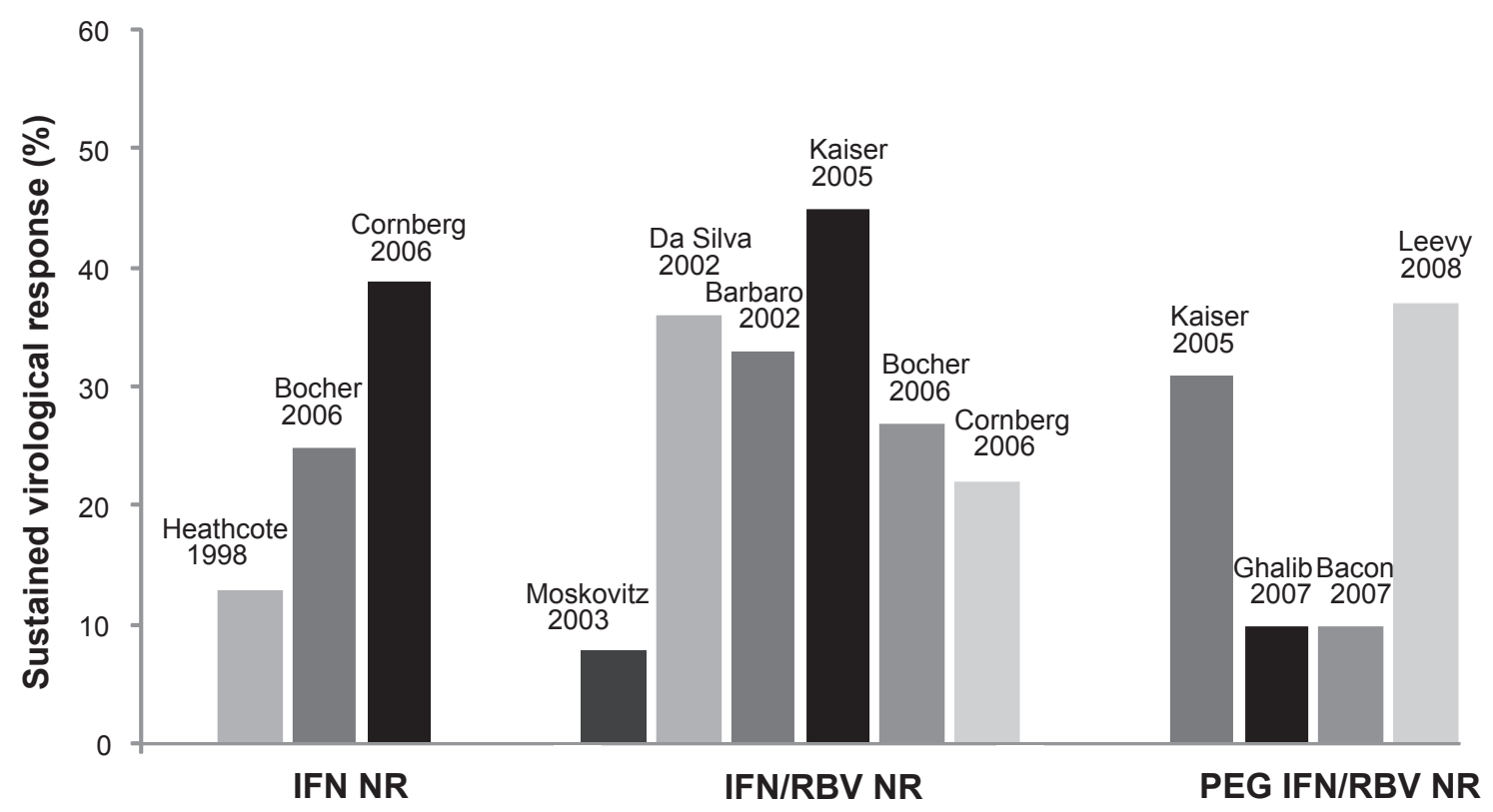

Figure 2 Clinical studies of consensus interferon in the retreatment of prior nonresponders to interferon-based therapy. ${ }^{2}$

${ }^{a}$ Note the studies by Heathcote et $\mathrm{a}^{33}$ Moskovitz et $\mathrm{al}^{35}$ and Barbaro et $\mathrm{al}{ }^{36}$ used consensus interferon as monotherapy. The remaining studies used combination consensus interferon and ribavirin.

Abbreviations: IFN, interferon; NR, nonresponder; PEG IFN, peginterferon; RBV, ribavirin. 
over a 72-week course in these patients. Prior nonresponders to PEG IFN and RBV are more challenging with retreatment and response rates may be significantly influenced by liver histology. Although one prospective study reported SVR rates as high as $31 \%$ in nonresponders to PEG IFN and $\mathrm{RBV}$ who are retreated with daily CIFN plus RBV, ${ }^{38}$ another retrospective study revealed a much lower response rate of $10 \%{ }^{44}$ This difference in SVR may be explained by the number of patients with bridging fibrosis or cirrhosis on liver biopsy, which was reported to be $28 \%$ versus $63 \%$ for each of these studies, respectively. In addition, the largest prospective trial to date evaluating the efficacy of daily CIFN and RBV in this population, known as the Daily-Dose Consensus Interferon and Ribavirin: Efficacy of Combined Therapy (DIRECT) Trial, appears to show no major improvement in SVR overall, although this trial involved a difficult-to-treat population characterized by a significant proportion of patients with advanced fibrosis. ${ }^{46}$

A novel approach utilizing CIFN therapy in nonresponders to combination PEG IFN and RBV has been described by Leevy in which patients who fail to achieve an EVR at 12 weeks of therapy on PEG IFN/RBV are converted to daily CIFN plus RBV with no wash-out period. ${ }^{47}$ Although a retrospective design was used in the selection of patients for this study, it provides an alternative strategy that may improve rates of SVR in an otherwise challenging group of patients with predominantly genotype 1 infection, high HCV RNA levels, and one-third African-American ethnicity. Patients who would otherwise discontinue therapy at 12 weeks due to failure to achieve an $\mathrm{EVR}^{7,14}$ completed an additional 48-week course of antiviral therapy consisting of RBV plus CIFN $15 \mu \mathrm{g}$ daily for 12 weeks followed by $15 \mu \mathrm{g} 3$ times weekly for 36 weeks. This 72 -week regimen was associated with an overall SVR of $37 \%$ in comparison with an SVR of approximately 11\%, which has been reported in EVR-negative patients who complete 72 weeks of PEG IFN and RBV. ${ }^{48}$

\section{The DIRECT trial}

The DIRECT trial is a multicenter, open-label, phase III study evaluating the efficacy of daily CIFN plus RBV in previous nonresponders to PEG IFN and RBV combination therapy. ${ }^{46}$ It is the largest prospective clinical trial involving the use of CIFN in this population to date. Although the DIRECT trial has yet to be published as a manuscript, the final results have been presented in abstract form. All patients enrolled in the study were considered nonresponders with less than a $2-\log _{10}$ decline in HCV RNA at 12 weeks during their previous course of PEG IFN plus RBV therapy or they had persistent viremia following at least 24 weeks of therapy. Patients were randomized to one of three treatment arms: CIFN $9 \mu \mathrm{g}$ daily plus RBV for 48 weeks; CIFN $15 \mu \mathrm{g}$ daily plus RBV for 48 weeks; or no treatment (Figure 3 ). Patients randomized to not receive treatment were eligible for a roll-over study at 24 weeks in which they could be randomized a second time to receive therapy as detailed in the treatment arms. Final data from 343 patients enrolled in the two treatment arms revealed overall SVR rates of $10 \%$ and $5 \%$ in the $15 \mu \mathrm{g}$ and $9 \mu \mathrm{g}$ groups, respectively. Subanalyses found that cirrhotics and prior null responders, defined by

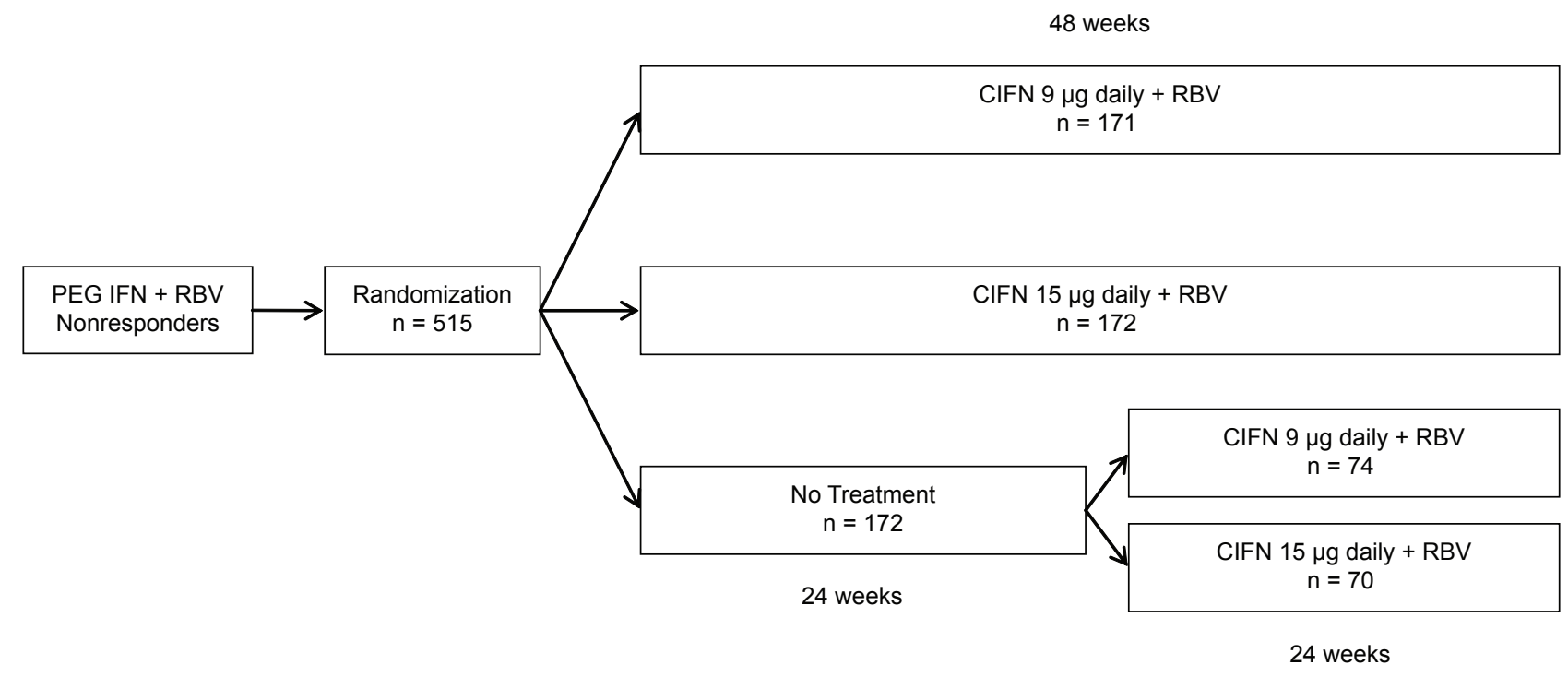

Figure 3 DIRECT trial protocol and randomization scheme. ${ }^{46}$

Abbreviations: CIFN, consensus interferon; DIRECT, Daily-Dose Consensus Interferon and Ribavirin: Efficacy of Combined Therapy; PEG IFN, peginterferon; RBV, ribavirin. 
inability to achieve a $2-\log _{10}$ decline in HCV RNA, had a significantly lower SVR rate. Noncirrhotic patients in the $15 \mu \mathrm{g}$ treatment arm who demonstrated at least a $2-\log _{10}$ decline in HCV RNA during prior therapy had the highest subgroup SVR at 29\%. The DIRECT trial was characterized by a predominantly difficult-to-treat population, which may in part explain the lower overall SVR compared with other studies evaluating CIFN-based therapy in prior PEG IFN/RBV nonresponders. The majority of patients in the DIRECT trial were described as having high HCV RNA levels, 79\% were considered null responders during previous treatment, 94\% had genotype 1 infection, 59\% had evidence of bridging fibrosis or cirrhosis on liver biopsy, and approximately $20 \%$ were of African-American ethnicity. Despite these challenges, the DIRECT trial most importantly identified potential subgroups of patients who have failed previous therapy and would benefit most from retreatment.

\section{Tolerability and adverse events}

Interferon-based therapy is known to be associated with significant side effects that may have an impact on tolerability, compliance, ability to maintain optimal dosing regimens, and ultimately response to therapy if dose reductions or discontinuations are required. As CIFN has been shown to have potent antiviral effects and has been studied with frequent dosing intervals up to once daily, the incidence of adverse events may be an important consideration in the retreatment of prior treatment failures. The side effect profile associated with CIFN is essentially the same as with other forms of IFN-based therapy. ${ }^{26}$ In addition, the incidence and frequency of adverse events during a course of combination CIFN and RBV therapy is similar to what has been reported in clinical trials of PEG IFN and RBV. ${ }^{6-8}$ The most common side effects that have been reported in clinical trials with CIFN include flu-like symptoms, gastrointestinal symptoms, rash, and depression. The frequency of these adverse events as noted in Table 2 include trials that involve daily dosing of CIFN up to $15 \mu \mathrm{g}$ per day and, in some cases induction doses up to $27 \mu \mathrm{g}$ per day. Based on these reported adverse events, there is no clear evidence that daily dosing of CIFN might result in an increase in side effects or a significant decline in tolerability as compared with weekly PEG IFN. The rates of discontinuation for adverse events and dose reductions in CIFN are also comparable to the rates reported with PEG IFN therapy.

\section{Conclusion}

Consensus interferon is a viable option in the retreatment of patients with chronic HCV who have failed prior IFN-based
Table 2 Most common adverse events associated with consensus interferon reported in clinical trials ${ }^{\mathrm{a}}$

\begin{tabular}{ll}
\hline Adverse event & Frequency (\%) \\
\hline Constitutional symptoms & $43-98$ \\
Flu-like symptoms & $21-36$ \\
Nausea & $10-32$ \\
Rash & $21-26$ \\
Depression & $22-25$ \\
Diarrhea & \\
Hematologic abnormalities & $16-29$ \\
Neutropenia & $6-29$ \\
Anemia & $4-18$ \\
Thrombocytopenia & \\
Dose reduction and discontinuation & $5-43$ \\
Dose reduction of CIFN for adverse event & $7-33$ \\
Discontinuation for adverse event &
\end{tabular}

ancludes studies with consensus interferon at subcutaneous doses up to $15 \mu \mathrm{g}$ daily for 48 weeks as well as induction doses up to $27 \mu \mathrm{g}$ daily. ${ }^{26,33,35-40,42-47}$

therapy and may have an important role for individuals who would otherwise be considered poor candidates for therapy based on a lower likelihood of achieving an SVR. Studies suggest that CIFN may have greater antiviral activity in vitro when compared to IFN- $\alpha$ and may have greater efficacy in difficult-to-treat populations, such as patients with high viral load and genotype 1 infection, and not have an increased incidence of adverse events compared with PEG IFN. Alternatively, CIFN requires more frequent dosing and potentially closer patient follow-up to ensure compliance. Many recent studies have utilized a daily dosing regimen of CIFN in order to provide more favorable kinetics and achieve maximal viral suppression during a course of therapy. Although the preferred dose of CIFN, duration of therapy, and timing of therapy in the setting of retreatment have not been well-established, daily weight-based RBV should be used in combination with CIFN in order to achieve optimal rates of SVR.

Several prospective clinical trials have identified patient populations who might benefit most from retreatment and have a greater potential for a successful course of therapy with CIFN. In light of these findings, clinicians should target these groups when considering potential candidates for retreatment. Patients who may particularly benefit from CIFN retreatment include prior nonresponders to standard IFN plus RBV as well as nonresponders and relapsers to prior PEG IFN plus RBV. These individuals otherwise have limited options as retreatment with conventional PEG IFN and RBV may be associated with low response rates. 
In addition, patients who achieved a significant degree of viral suppression during prior IFN-based therapy and patients with early stages of fibrosis have a greater likelihood of achieving a SVR with CIFN retreatment. The ability to achieve significant viral suppression during a previous course of IFN-based therapy has been shown to be a predictor of successful response in several studies involving CIFN, $34,40,46,47$ and has also been described in retreatment regimens that include PEG IFN. ${ }^{15,16,18,21,22}$ Liver histology also appears to have a significant impact on virologic response associated with CIFN therapy, as patients who have not developed advanced fibrosis are more likely to achieve SVR with retreatment. ${ }^{46}$

Interpretation of the various clinical studies involving use of CIFN for prior therapy failures in chronic HCV infection can be challenging due to wide variations in study design, dosing regimens, duration of therapy, and patient populations. These studies are limited by differences in dosing and frequency of CIFN, inclusion of induction doses of CIFN at times without the use of RBV, and heterogeneous populations based on prior response to therapy. In addition, comparative data between CIFN and conventional IFN-based regimens is limited. ${ }^{35,45}$ Overall, these factors challenge our ability to determine whether CIFN-based regimens may indeed be superior to PEG IFN and RBV in the retreatment of HCV-infected individuals who failed prior IFN-based therapy. Despite these limitations, it is clear that some patients with chronic HCV may benefit from retreatment with CIFN. Individualized therapy targeting specific patient groups will be an important consideration in successful management of these patients. Additional prospective studies of CIFN are needed to identify optimal treatment strategies as well as establish which populations will benefit most from retreatment.

\section{Abbreviations}

CIFN, consensus interferon; DIRECT, Daily-Dose Consensus Interferon and Ribavirin: Efficacy of Combined Therapy; EVR, early virologic response; HCC, hepatocellular carcinoma; HCV, hepatitis $\mathrm{C}$ virus; IFN, interferon; JAK-STAT, Janus kinase-signal transducer and activator of transcription; MU, million units; NR, nonresponders; PEG IFN, peginterferon; QD, daily; QOD, every other day; $\mathrm{RBV}$, ribavirin; SC, subcutaneous; SVR, sustained virologic response; TIW, 3 times weekly.

\section{Disclosures}

The authors disclose no conflicts of interest.

\section{References}

1. Bell BP, Manos MM, Zaman A, et al. The epidemiology of newly diagnosed chronic liver disease in gastroenterology practices in the United States: results from population-based surveillance. Am J Gastroenterol. 2008;103(11):2727-2736.

2. Seeff LB. Natural history of chronic hepatitis C. Hepatology. 2002;36(5 Suppl 1):S35-S46.

3. Poynard T, McHutchison J, Manns M, et al. Impact of pegylated interferon alfa-2b and ribavirin on liver fibrosis in patients with chronic hepatitis C. Gastroenterology. 2002;122(5):1303-1313.

4. Craxi A, Camma C. Prevention of hepatocellular carcinoma. Clin Liver Dis. 2005;9(2):329-346, viii.

5. Maylin S, Martinot-Peignoux M, Moucari R, et al. Eradication of hepatitis $\mathrm{C}$ virus in patients successfully treated for chronic hepatitis $\mathrm{C}$. Gastroenterology. 2008;135(3):821-829.

6. Manns MP, McHutchison JG, Gordon SC, et al. Peginterferon alfa-2b plus ribavirin compared with interferon alfa- $2 b$ plus ribavirin for initial treatment of chronic hepatitis C: a randomised trial. Lancet. 2001;358(9286):958-965.

7. Fried MW, Shiffman ML, Reddy KR, et al. Peginterferon alfa-2a plus ribavirin for chronic hepatitis C virus infection. $N$ Engl J Med. 2002;347(13):975-982.

8. Hadziyannis SJ, Sette H Jr, Morgan TR, et al. Peginterferon-alpha2a and ribavirin combination therapy in chronic hepatitis $\mathrm{C}$ : a randomized study of treatment duration and ribavirin dose. Ann Intern Med. 2004;140(5):346-355.

9. Muir AJ, Bornstein JD, Killenberg PG. Peginterferon alfa-2b and ribavirin for the treatment of chronic hepatitis $\mathrm{C}$ in blacks and non-Hispanic whites. N Engl J Med. 2004;350(22):2265-2271.

10. Hoofnagle JH, Mullen KD, Jones DB, et al. Treatment of chronic non-A,non-B hepatitis with recombinant human alpha interferon. A preliminary report. $N$ Engl J Med. 1986;315(25):1575-1578.

11. Poynard T, Marcellin P, Lee SS, et al. Randomised trial of interferon alpha2b plus ribavirin for 48 weeks or for 24 weeks versus interferon alpha2b plus placebo for 48 weeks for treatment of chronic infection with hepatitis $\mathrm{C}$ virus. International Hepatitis Interventional Therapy Group (IHIT). Lancet. 1998;352(9138):1426-1432.

12. McHutchison JG, Gordon SC, Schiff ER, et al. Interferon alfa-2b alone or in combination with ribavirin as initial treatment for chronic hepatitis C. Hepatitis Interventional Therapy Group. $N$ Engl J Med. 1998;339(21):1485-1492.

13. Strader DB, Wright T, Thomas DL, Seeff LB: Diagnosis, management, and treatment of hepatitis C. Hepatology. 2004;39(4):1147-1171.

14. Davis GL, Wong JB, McHutchison JG, Manns MP, Harvey J, Albrecht J. Early virologic response to treatment with peginterferon alfa-2b plus ribavirin in patients with chronic hepatitis C. Hepatology. 2003;38(3):645-652.

15. Shiffman ML, Di Bisceglie AM, Lindsay KL, et al. Peginterferon alfa-2a and ribavirin in patients with chronic hepatitis $\mathrm{C}$ who have failed prior treatment. Gastroenterology. 2004;126(4):1015-1023.

16. Jacobson IM, Gonzalez SA, Ahmed F, et al. A randomized trial of pegylated interferon alpha-2b plus ribavirin in the retreatment of chronic hepatitis C. Am J Gastroenterol. 2005;100(11):2453-2462.

17. Mathew A, Peiffer LP, Rhoades K, McGarrity T. Sustained viral response to pegylated interferon alpha-2b and ribavirin in chronic hepatitis C refractory to prior treatment. Dig Dis Sci. 2006;51(11):1956-1961.

18. Sherman M, Yoshida EM, Deschenes M, et al. Peginterferon alfa-2a (40 KD) plus ribavirin in chronic hepatitis $C$ patients who failed previous interferon therapy. Gut. 2006;55(11):1631-1638.

19. Krawitt EL, Ashikaga T, Gordon SR, Ferrentino N, Ray MA, Lidofsky SD. Peginterferon alfa-2b and ribavirin for treatmentrefractory chronic hepatitis C. J Hepatol. 2005;43(2):243-249.

20. Basso M, Torre F, Grasso A, et al. Pegylated interferon and ribavirin in re-treatment of responder-relapser HCV patients. Dig Liver Dis. 2007;39(1):47-51. 
21. Moucari R, Ripault MP, Oules V, et al. High predictive value of early viral kinetics in retreatment with peginterferon and ribavirin of chronic hepatitis $\mathrm{C}$ patients non-responders to standard combination therapy. J Hepatol. 2007;46(4):596-604.

22. Taliani G, Gemignani G, Ferrari C, et al. Pegylated interferon alfa-2b plus ribavirin in the retreatment of interferon-ribavirin nonresponder patients. Gastroenterology. 2006;130(4):1098-1106.

23. Gale M Jr, Foy EM. Evasion of intracellular host defence by hepatitis C virus. Nature. 2005;436(7053):939-945.

24. van Pesch V, Lanaya H, Renauld JC, Michiels T. Characterization of the murine alpha interferon gene family. J Virol. 2004;78(15): 8219-8228.

25. Blatt LM, Davis JM, Klein SB,Taylor MW. The biologic activity and molecular characterization of a novel synthetic interferon-alpha species, consensus interferon. J Interferon Cytokine Res. 1996;16(7): 489-499.

26. Infergen $^{\circledR}$ (Interferon alfacon-1) [package insert]. Revised July 27, 2006. Valeant Pharmaceuticals. Costa Mesa, CA, USA.

27. Fattovich G, Zagni I, Minola E, et al. A randomized trial of consensus interferon in combination with ribavirin as initial treatment for chronic hepatitis C. J Hepatol. 2003;39(5):843-849.

28. Rustgi VK, Bonkovsky HL, Arora S, Zeuzem SP, Manns M, Blatt LM: Daily versus three-times-weekly IFN Alfacon-1 in previously untreated HCV patients results in a significantly greater rate of SVR: Final results of an international, phase 4 study [abstract S1541]. Gastroenterology. 2005;128(4 Suppl 2):A-716.

29. Tong MJ, Reddy KR, Lee WM, et al. Treatment of chronic hepatitis C with consensus interferon: a multicenter, randomized, controlled trial. Consensus Interferon Study Group. Hepatology. 1997;26(3):747-754.

30. Jensen DM, Krawitt EL, Keeffe EB, et al. Biochemical and viral response to consensus interferon (CIFN) therapy in chronic hepatitis $\mathrm{C}$ patients: effect of baseline viral concentration. Consensus Interferon Study Group. Am J Gastroenterol. 1999;94(12):3583-3588.

31. Sjogren MH, Sjogren R, Holtzmuller K, et al. Interferon alfacon-1 and ribavirin versus interferon alpha- $2 \mathrm{~b}$ and ribavirin in the treatment of chronic hepatitis C. Dig Dis Sci. 2005;50(4):727-732.

32. Sjogren MH, Sjogren R, Jr, Lyons MF, et al. Antiviral response of HCV genotype 1 to consensus interferon and ribavirin versus pegylated interferon and ribavirin. Dig Dis Sci. 2007;52(6):1540-1547.

33. Heathcote EJ, Keeffe EB, Lee SS, et al. Re-treatment of chronic hepatitis C with consensus interferon. Hepatology. 1998;27(4):1136-1143.

34. Heathcote EJ, James S, Mullen KD, Hauser SC, Rosenblate H, Albert DG Jr. Chronic hepatitis $\mathrm{C}$ virus patients with breakthroughs during interferon treatment can successfully be retreated with consensus interferon. The Consensus Interferon Study Group. Hepatology. 1999;30(2):562-566.

35. Miglioresi L, Bacosi M, Russo F, et al. Consensus interferon versus interferon-alpha $2 \mathrm{~b}$ plus ribavirin in patients with relapsing $\mathrm{HCV}$ infection. Hepatol Res. 2003;27(4):253-259.
36. Barbaro G, Barbarini G: Consensus interferon for chronic hepatitis $\mathrm{C}$ patients with genotype 1 who failed to respond to, or relapsed after, interferon alpha-2b and ribavirin in combination: an Italian pilot study. Eur J Gastroenterol Hepatol. 2002;14(5):477-483.

37. Moskovitz DN, Manoharan P, Heathcote EJ: High dose consensus interferon in nonresponders to interferon alpha-2b and ribavirin with chronic hepatitis C. Can J Gastroenterol. 2003;17(8):479-482.

38. Kaiser S, Hass HG,Gregor M. Successful retreatment of interferon/ ribavirin nonresponders with daily dosing of consensus interferon [abstract 570]. J Hepatol. 2005;42 Suppl 2:207-208.

39. Bocher WO, Schuchmann M, Link R, et al. Consensus interferon and ribavirin for patients with chronic hepatitis $\mathrm{C}$ and failure of previous interferon-alpha therapy. Liver Int. 2006;26(3):319-325.

40. Cornberg M, Hadem J, Herrmann E, et al. Treatment with daily consensus interferon (CIFN) plus ribavirin in non-responder patients with chronic hepatitis C: a randomized open-label pilot study. J Hepatol. 2006;44(2):291-301

41. Layden TJ, Layden JE, Reddy KR, Levy-Drummer RS, Poulakos J, Neumann AU: Induction therapy with consensus interferon (CIFN) does not improve sustained virologic response in chronic hepatitis C. J Viral Hepat. 2002;9(5):334-339.

42. Alaimo G, Di Marco V, Ferraro D, et al. Different doses of consensus interferon plus ribavirin in patients with hepatitis $\mathrm{C}$ virus genotype 1 relapsed after interferon monotherapy: a randomized controlled trial. World J Gastroenterol. 2006;12(42):6861-6864.

43. da Silva LC, Bassit L, Ono-Nita SK, et al. High rate of sustained response to consensus interferon plus ribavirin in chronic hepatitis $\mathrm{C}$ patients resistant to alpha-interferon and ribavirin: a pilot study. J Gastroenterol. 2002;37(9):732-736.

44. Ghalib RH, Levine CD, Friedman DA, Rashdan S, Schwartz AG, Weinstein J. Consensus interferon plus ribavirin therapy in patients who are nonresponders or relapsers to prior PEG IFN plus ribavirin [abstract M1874]. Gastroenterology. 2007;132(4 Suppl 2):A-794.

45. Kaiser S, Lutze B, Sauter B, et al. Retreatment of HCV genotype 1 relapse patients to peginteferon/ribavirin therapy with an extended treatment regimen of 72 weeks with consensus interferon/ribavirin versus peginterferon alpha/ribavirin [abstract 1310]. Hepatology. 2007;46(4 Suppl 1):819A.

46. Bacon B, Regev A, Ghalib RH, et al. The DIRECT trial (Daily-Dose Consensus Interferon and Ribavirin: Efficacy of Combined Therapy): Treatment of non-responders to previous pegylated interferon plus ribavirin: Sustained virologic response data [abstract 168]. Hepatology. 2007;46(4 Suppl 1):311A-312A.

47. Leevy CB. Consensus interferon and ribavirin in patients with chronic hepatitis $\mathrm{C}$ who were nonresponders to pegylated interferon alfa- $2 \mathrm{~b}$ and ribavirin. Dig Dis Sci. 2008;53(7):1961-1966.

48. Berg T, von Wagner M, Nasser S, et al. Extended treatment duration for hepatitis $\mathrm{C}$ virus type 1: comparing 48 versus 72 weeks of peginterferonalfa-2a plus ribavirin. Gastroenterology. 2006;130(4):1086-1097. 